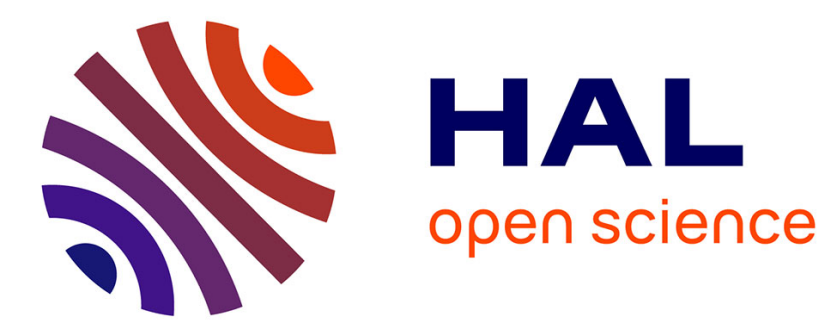

\title{
Histoire des démarches en sciences et épistémologie scolaire \\ Jean-Yves Cariou
}

\section{To cite this version:}

Jean-Yves Cariou. Histoire des démarches en sciences et épistémologie scolaire . RDST - Recherches en didactique des sciences et des technologies , 2011, 3, pp.83-106. hal-01535167

\section{HAL Id: hal-01535167 https://hal.univ-antilles.fr/hal-01535167}

Submitted on 8 Jun 2017

HAL is a multi-disciplinary open access archive for the deposit and dissemination of scientific research documents, whether they are published or not. The documents may come from teaching and research institutions in France or abroad, or from public or private research centers.
L'archive ouverte pluridisciplinaire HAL, est destinée au dépôt et à la diffusion de documents scientifiques de niveau recherche, publiés ou non, émanant des établissements d'enseignement et de recherche français ou étrangers, des laboratoires publics ou privés. 


\title{
Histoire des démarches en sciences et épistémologie scolaire
}

\author{
RDST - Recherches en Didactique des Sciences et des Technologies n³, 2011, p. 83-106.
}

\section{Jean-Yves Cariou}

Université des Antilles et de la Guyane

\begin{abstract}
Divers auteurs ont relevé l'existence d'une épistémologie «spontanée » chez les enseignants, de nature empiriste et inductiviste, conforme à une certaine vision de l'histoire des sciences et de la pratique de la recherche scientifique. Le didacticien, s'il estime utile de tenter de déconstruire cette vision et d'en faire reconstruire une autre aux enseignants, afin que ceux-ci modifient dans ce sens leurs pratiques au nom d'une plus grande conformité avec le déroulement de la recherche scientifique, doit pouvoir justifier cette tentative, en s'appuyant sur les travaux des historiens des sciences et des épistémologues. Il lui sera dans le même temps utile de discerner certains des éléments qui sont à l'origine d'une conception qu'il juge erronée, ce que peut permettre, au moins partiellement, une analyse de l'impact des discours sur la méthode en sciences. Ces éléments permettent de dégager des pistes pour la formation des enseignants et des élèves.
\end{abstract}

L'appel à un appui sur l'histoire des sciences pour l'enseignement scientifique est ancien : « le savoir qui serait donné comme un fil à dévider encore, il faudrait le transmettre et le faire comprendre si possible de la manière même dont il a été découvert », proclame Bacon dès 1605 (p. 185). Bacon, dont les discours sur la méthode $(1605,1620)$ précèdent celui de Descartes (1637), préconise une approche historique qui «encourage à examiner » et permet « une investigation [inquiry] » : il reliait déjà, dans l'un des premiers textes accompagnant la révolution scientifique du début du XVIIe siècle, histoire des découvertes, enseignement et investigation.

Trois siècles plus tard, lors de la réforme de 1902 en France, la "règle épistémologique" qui prévaut est qu'il convient de "procéder comme la science expérimentale elle-même » (Kahn, 2002, p. 105). Certes, mais comment procède-t-elle ? Les textes officiels qui tentent d'extraire de l'histoire des sciences des éléments de méthodologie à l'usage des enseignants le font de manière souvent critiquable. Dès cette époque, Duhem, physicien et historien des sciences, relevait -en la déplorant- l'influence de la "méthode newtonienne" dans l'enseignement secondaire (1906, p. 305). Procéder comme la science elle-même serait procéder comme Newton le prétendait : cette coloration empiriste et inductiviste persiste de nos jours dans les classes. Les enseignants d'alors, comme ceux d'aujourd'hui, n'omettent pas de narrer la célébrissime histoire de la pomme, qui se conforme si bien à une démarche dans laquelle les théories sont extraites des "faits". Connaissant peu l'histoire des sciences, ils prennent de tels exemples comme caractéristiques de la démarche scientifique. "C'est la science expérimentale des instructions ministérielles », résume le professeur de physique Bachelard : « voir pour comprendre, tel est l'idéal de cette étrange pédagogie » $(1932$, p. 12).

La force de l'enracinement d'une telle conception chez les enseignants et l'obstacle que cela constitue pour la mise en œuvre de démarches plus en conformité avec les itinéraires des scientifiques au laboratoire ont régulièrement été signalés (Johsua \& Dupin, 1993, p. 216219 ; Robardet \& Guillaud, 1997 ; Clément, 1998 ; Rumelhard, 2000 ; Astolfi, 2002 ; Apostolou \& Koulaidis, 2010). 
L'analyse de l'histoire des méthodes et des discours sur les méthodes en sciences paraît alors utile au didacticien pour mieux cerner l'origine possible de cette conception et lui permettre d'envisager un environnement favorable pour tenter de la déconstruire, ou, au moins, pour amener les enseignants à la relativiser dans leurs pratiques. Nous proposons ainsi de jeter un regard sur les discours historiques sur les méthodes, permettant d'envisager un rapport critique à l'histoire des sciences. Nous dégagerons le lourd impact du discours de Newton et de ses adeptes sur l'enseignement des sciences, avant de voir comment les avancées épistémologiques ont pu, au $\mathrm{XX}^{\mathrm{e}}$ siècle, armer les didacticiens dans leurs efforts, malheureusement relativement infructueux face à une tradition épistémologique très enracinée. Ce qui nous conduira à envisager des approches didactiques tenant compte de ces éléments.

\section{L'arrière-plan historique de l'épistémologie «spontanée » des enseignants}

\subsection{Constats en didactique}

L'épistémologie, souligne Dorier (2000), « apparaît comme le terme médiateur qui fait le lien entre le travail historique et le travail didactique ». Les références aux données épistémologiques sur la manière dont se construit la connaissance accompagnèrent les premières recherches en didactique (Host, 1998). L'analyse des mécanismes de l'élaboration scientifique par les épistémologues reposant à son tour sur des études historiques, les travaux des historiens des sciences constituent une assise pour la recherche en didactique. Dans certains cas, des didacticiens ont été engagés eux-mêmes dans des recherches historiques, ainsi Host entreprit-t-il une thèse d'histoire des sciences sous la direction de Canguilhem, et Gohau (élève du même Canguilhem), professeur de lycée doublé d'un historien des sciences de renom, n'a-t-il cessé de transpercer de ses flèches épistémologiques les égarements méthodologiques de l'enseignement des sciences.

La vision classique qu'ont les enseignants de la science est souvent qualifiée de conception «empiriste» et «inductiviste ». Empiriste parce que la découverte partirait de faits perceptibles indépendamment d'idées préconçues et de théories ; inductiviste car de tels faits constitueraient un gisement duquel extraire les concepts. D'où la présentation initiale d'observations ou d'expériences aux élèves, afin qu'en découle la "mise en évidence", terme fort approprié, des notions au programme. Un "présupposé inductiviste", disent Johsua et Dupin, qui constitue «le credo largement prédominant» (1993, p. 216-219), une "épistémologie spontanée" pour Demounem et Astolfi (1996, p. 99) comme pour Rumelhard qui dénonce la pédagogie de l'évidence (2000). Astolfi parlait d'une «norme scolaire (...) qui impose la méthode inductive »(2002). Clément (1998), résumant dix ans de recherche en didactique de la biologie, confirme cet aspect, tout comme pour la physique Robardet et Guillaud (1997, p. 44), qui consacrent notamment un chapitre à l'inductivisme dans l'enseignement scientifique, "épistémologie implicite", et notent que de nombreuses études effectuées tant en France qu'à l'étranger confirment ce diagnostic.

Plus récemment, le rapport sur L'enseignement des sciences en Europe ${ }^{1}$ (2006, p. 58-65), confirme : «Dans l'enseignement secondaire, les expériences sont principalement utilisées dans une perspective d'illustration des concepts, de vérification d'une loi, ou dans une démarche inductiviste (...). L'élève est alors placé en situation d'exécuter des manipulations qui lui sont prescrites, (...) les conclusions semblant s'imposer d'elles-mêmes, lorsqu'elles ne sont pas connues d'avance. » Gil-Pérez et al. (2005) choisissent même de parler, fort significativement, d'absence de travail expérimental en classe de science lorsque les seules

\footnotetext{
${ }^{1}$ L'enseignement des sciences dans les établissements scolaires en Europe. État des lieux des politiques et de la recherche. Eurydice, 2006.
} 
expériences qui s'y déroulent sont le fait d'élèves simples exécutants. L'étude récente de Apostolou et Koulaidis (2010) indique toujours la persistance d'une position "empiricoinductiviste" dominante chez les enseignants scientifiques. Donner une image plus authentique de la nature de la science (concept abrégé par les anglo-saxons en NOS, nature of science) devient alors un objectif primordial (Lederman, 2007).

Gohau (1977) estime que «dans l'ignorance où est l'enseignant des sciences de l'histoire et de l'épistémologie de sa discipline, les techniques inductives ont abouti à des absurdités en croyant qu'il suffisait de retourner la méthode traditionnelle déductive pour fonder une pédagogie active de découverte. » $\mathrm{Si}$ l'inductivisme est tant réclamé comme "règle épistémologique" par les réformateurs de 1902, c'est en effet que beaucoup d'enseignants, devant la masse de notions à transmettre, recouraient à l'exposé dogmatique de connaissances théoriques, livresques, avant de montrer ou d'évoquer des faits qui s'en déduisent.

Cette opposition si nette entre déduction et induction en 1902 récapitule, en quelque sorte, une opposition de bien plus longue haleine dans l'histoire des sciences.

\subsection{Voies d'accès à la connaissance dans l'histoire des sciences}

Bacon souhaite que le professeur suive la voie du savant pour «adhérer à la moelle des choses » (1620, p. 78) et monter progressivement, à partir d'expériences et d'observations, vers les axiomes élevés : voie inductive, lente, mais sûre. Elle ne satisfait pas Descartes, pour qui l'expérience est souvent trompeuse, et toutes les idées douteuses. Il n'en conserve qu'une poignée, "évidentes", et à partir de ce seul ancrage fiable il veut n'admettre que des certitudes enchaînées par les maillons d'acier de la déduction. De ce bastion inexpugnable, il explique le monde, avançant des hypothèses, des "causes imaginées", dont il dit toutes les raisons de ne pas douter. La tradition oppose les deux hommes, en caricaturant leur position : Bacon n'arrivant à l'idée qu'au sommet d'une pyramide de faits, Descartes les scrutant de haut pour ne les toucher qu'au bout de ses raisonnements, et négligeant l'expérience.

Mais Bacon récuse les empiristes-fourmis qui amassent vainement sans s'élever, autant que la spéculateurs-araignées qui tissent leur toile dans les airs. Lui est abeille, qui récolte et construit (1620, p. 157). Il veut coller aux faits en évitant les envols intempestifs de l'esprit. Mais, loin de rejeter toute hypothèse, il en considère explicitement l'usage lorsque son ascension inductive le conduit à un carrefour de possibles $(1620$, p. 255) : il formule alors diverses hypothèses et imagine une expérience pour trancher.

Descartes, lui, est dépeint sous les traits de l'araignée, dont on ne se privera pas d'agiter la toile : Kant, dans sa critique d'une raison trop pure, souligne que «quand on est hors du cercle de l'expérience, on est certain de ne pas être contredit par elle » (1787, p. 36).

Mais Descartes écrivait qu'en partant des principes, un effet pouvait « être déduit en plusieurs diverses façons », et devant ces chemins multiples, il doit pareillement «chercher derechef quelques expériences » (1637, p. 101). Si sa méthode reste avant tout déductive, il n'hésite pas à ne voir dans ses déductions, si plusieurs se valent, que des hypothèses à tester par l'expérience. Descartes, faisant un crochet par l'expérience, rejoint alors Bacon, qui fait un détour par l'hypothèse, sur la même troisième voie, hypothético-déductive.

Or celle-ci n'est pas déserte : sans faire primer les considérations méthodologiques, mais loin d'en être dépourvus, Kepler, Galilée, Harvey l'explorent déjà. Kepler aime entraîner son lecteur dans son itinéraire épineux : «Lecteur, (...) il faut que tu traverses auparavant les mêmes broussailles que celles entre lesquelles je me suis faufilé $»^{2}$. Harvey relate sa découverte du trajet circulaire du sang (1628) en exposant la soumission de ses hypothèses à l'épreuve d'expériences.

\footnotetext{
${ }^{2}$ Paralipomènes à Vitellion (1604, IV, 2), Astronomia pars Optica, tr. Catherine Chevalley, Vrin, 1980.
} 
Dans les années 1670-80, Rohault, chef de file des cartésiens, et Hooke, disciple de Bacon, convergent dans une description claire de la fructueuse voie hypothético-déductive. Rohault distingue nettement l'expérience commune, l'expérience pour voir et l'expérience de mise à l'épreuve, qui «peut faire découvrir la vérité ou la fausseté des opinions » (1671, préface). Sur Hooke, l'historien de la géologie Ellenberger note (1994, p. 90) : «Il fait un remarquable exposé épistémologique sur la Méthode à mettre en œuvre dans l'étude de la Nature, texte encore à méditer ».

Entre la thèse déductiviste et l'antithèse inductiviste, la synthèse hypothético-déductive semble devoir s'étendre sur les sciences. Paraît alors un jeune mathématicien qui pense pouvoir porter en physique une certitude égale à celle de son domaine d'origine : Newton.

\subsection{Newton et la querelle des hypothèses}

Il explique d'abord la lumière (1672), mettant en avant une expérience cruciale d'où il tire des conclusions qui selon lui s'ensuivent nécessairement des phénomènes. Une analyse moderne montrera qu'il s'agit d'une reconstruction (Kuhn, 1958 ; Blay, 2006). La lumière, Hooke en est le spécialiste, avec Huygens : il s'agit, pour eux, d'une onde. Newton parle de corpuscules. On lui demande de rendre compte de ses hypothèses, lui qui se voit n'avancer que des vérités. On lui oppose d'autres hypothèses. On lui en propose. Hooke lui reproche d'utiliser les siennes sans le citer. L'orgueil de Newton est atteint. Une entrée en scène rude, mais qui s'apaise bientôt : Newton répond à un Hooke conciliant « Si j'ai vu plus loin, c'est en me tenant sur les épaules de géants », que sont Descartes et, justement, Hooke.

L'hiver 1679-1680, Hooke lui demande son avis sur ses hypothèses personnelles concernant la gravitation : pour lui, les astres sont non pas repoussés par une force décrite par Descartes et que Huygens nomme centrifuge, mais mus par «l'inflexion d'un mouvement direct en une courbe par l'effet d'un principe attractif »-qui empêche l'astre sur son orbite, au sens propre, de "prendre la tangente". Et, seconde hypothèse: cette force doit être inversement proportionnelle au carré de la distance. Rien de moins.

Adoptant la vision de Hooke, Newton cesse de parler d'une force centrifuge, lui substituant une force qu'il nomme centripète. Il passe, en quelque sorte, de l'épaule du premier géant qu'il a désigné (Descartes) à celle du second (Hooke).

Mais Newton, qui prétendra par la suite avoir découvert de la loi de l'attraction dès 1666 , son regard allant d'une pomme à la Lune, répond à Hooke qu'il ne s'intéresse pas à ces questions. Or lorsqu'en 1686 il est prêt à publier, Hooke demande que l'apport de ses hypothèses soit reconnu : le refus est catégorique. "Je le savais déjà", dit-il en substance, "je ne m'en suis pas servi”, "d'ailleurs ces idées sont dans un paragraphe d'une lettre que j'ai écrite en 1673 à Huygens". Et "s'il en est ainsi, je ne publie pas".

La lettre de 1673 sera retrouvée au XIX ${ }^{\mathrm{e}}$ siècle dans les archives de Huygens, mais sans le paragraphe en question (Drake, 1996, p. 33). Certains auteurs pensent que l'histoire de la pomme, qui n'apparaît qu'en 1726, est une invention pour ne pas reconnaître l'utilisation des hypothèses de Hooke. Newton place cet événement en 1666 : Guicciardini (2003, p. 29) se demande pourquoi alors il soutient en 1675 une théorie de la gravitation sans attraction, et Kollerstrom (1999) relève qu'il interprète toujours la comète de 1680 avec une simple adaptation de l'idée de Descartes.

La querelle avec Hooke est fondamentale non seulement par ses aspects conceptuels, mais également d'un point de vue méthodologique. I. B. Cohen, spécialiste et traducteur de Newton, commente : «L'analyse de Hooke contient la clé du problème des mouvements 
célestes. Elle fut un élément capital pour l'élaboration de la mécanique céleste tel que Newton l'exposa dans ses Principia » (1960, p. 243).

L'analyse de Hooke, ce sont ses hypothèses ingénieuses, ces sortes d'envolées qui caractérisaient aussi les cogitations de Descartes. Mais il était bien plus aisé, pour Newton, de se débarrasser -après les avoir longtemps adoptés- des "tourbillons" imaginaires du monde cartésien que des hypothèses révolutionnaires sur lesquelles Hooke l'interroge directement. Les ombres de ces deux géants, décidément, ne cessent de se dresser derrière Newton, qui va s'employer à les estomper.

Descartes présentait son système du monde dans ses Principes de la Philosophie (1644) : Newton le détruit et y substitue le sien dans son propre ouvrage, dont le titre fort semblable comporte les mêmes termes, rehaussés de deux qualificatifs : Principes Mathématiques de la Philosophie Naturelle (1687).

Newton triomphant impose ses vues : en 1713, il insère dans la seconde édition des Principes cette fameuse phrase que Hooke, mort entretemps, ne lira pas : hypotheses non fingo, « je ne fais pas d'hypothèses ", sentence qui fit couler beaucoup d'encre. Duhem (1906, p. 67) et Koyré (1968, p. 60) traduisent «je ne feins pas » (plus conforme au latin), feindre impliquant la fausseté. La formule venant peu après le rejet de l'hypothèse des tourbillons, on peut relever que l'emploi de non fingo paraît dirigé contre un terme qu'utilise fréquemment Descartes dans ses propres Principes : «Feignons donc [fingamus] que cette Terre... » (IV, $\S 2$ ), «nous pouvons feindre à plaisir [effingere] plusieurs idées (...) que nous feignons [effinximus] quand bon nous semble » $(\mathrm{I}, \S 16)$.

Non fingo contre fingamus: si Newton vise les fictions cartésiennes, il poursuit, après sa formule, en ne laissant échapper aucune sorte d'hypothèse :

«Tout ce qui n'est pas déduit des phénomènes doit être appelé hypothèse et les hypothèses, qu'elles soient métaphysiques, physiques, se rapportant aux qualités occultes ou mécaniques, n'ont pas de place en philosophie expérimentale. En cette philosophie, les propositions sont déduites des phénomènes et rendues générales par induction. » (1713, p. 179).

Les propos de Newton ont entraîné un large rejet des hypothèses, puisque tel était l'enseignement professé par celui à qui tout obéissait, sur la Terre comme aux cieux : les mouvements des planètes et des satellites, les marées, la forme de la Terre et jusqu'à la chute des pommes... L'Encyclopédie décrit le souci de Newton et de ses disciples de les «proscrire entièrement »: "ils se sont élevés contre les hypothèses, ils ont tâché de les rendre suspectes et ridicules, en les appelant le poison de la raison et la peste de la philosophie » (article Hypothèse, 1765).

Certains auteurs ont avant tout retenu le rejet d'hypothèses métaphysiques, tels Comte qui s'appuie sur Newton pour chasser de la science les fantômes métaphysiques qui y rôdent encore : «Il y a, sans doute, beaucoup d'analogie entre ma philosophie positive et ce que les savants anglais entendent, depuis Newton surtout, par philosophie naturelle. » (1830, p. VII). Ainsi, pour la gravitation, s'en tiendra-t-on à la loi, sans se perdre dans ce que pourrait être sa cause première.

Comte cependant, loin de condamner l'usage des hypothèses, insiste sur leur vérification, et épingle pour le non-respect de ses propres préceptes ce Newton «dont l'exclamation favorite était : Ô physique! Garde-toi de la métaphysique! ", et qui s’y laisse cependant entraîner (1835, p. 639). Duhem (1906) voit dans le précepte newtonien une volonté de s'en tenir à une représentation condensée des phénomènes, sans recherche illusoire des causes, "prudente réserve » (p. 69), tout en critiquant la prétendue méthode inductive dénuée d'hypothèses de Newton et de son fidèle adepte Ampère (p. 289-305). Koyré, qui analyse «l'aversion de 
Newton pour les hypothèses " et l'utilisation qu'il en fait pourtant tout en la dénonçant chez les autres, conclut que le mot "hypothèse" semble être devenu pour lui « un de ces termes curieux tels, par exemple, que celui d'hérésie, que nous n'appliquons jamais à nous-mêmes, mais seulement à d'autres » (1968, p. 53-84). Toutes les sciences, note-t-il, tentent alors de « se conformer au modèle newtonien de la connaissance empirico-déductive et de s'en tenir aux lois formulées par Newton» (p. 39). Une situation que résume bien Medawar : «Deux cent ans après Newton, personne ne pouvait avouer faire usage d'hypothèses sans jeter un coup d'œil plein de malaise derrière lui. » (1967, p. 140).

Tel est le coup d'arrêt newtonien : il bannit les hypothèses de la science, disperse à jamais celles de Descartes, escamote celles de Hooke dans la foulée et ne veut plus entendre parler que d'induction. La "claque de Newton" aux hypothèses barre durablement la troisième voie, et la démarche inductive règne alors sans partage : le monde scientifique mettra près de deux siècles à s'en remettre.

Le système de Descartes, qui avait tant séduit et faisait se pâmer les Femmes savantes de Molière, bâti sur des causes imaginées, s'effondre ; le nouveau système, fondé selon Newton sur l'induction, est extraordinaire: il explique aussi le monde, mais en plus, il marche. Newton triomphe, et avec lui, ses préceptes méthodologiques.

\subsection{L'aube après la longue nuit des hypothèses}

Pour les hypothèses, il n'y aura pas de plaidoyer influent avant les années 1830-40, même si certains brandissent un flambeau notable tels Diderot. Ce n'est qu'après une mise à l'écart quasi générale des discours qui aura duré quelque 150 ans (Laudan, 1981, p. 9-11), que la méthode de l'hypothèse commence à trouver des défenseurs parmi ceux qui relèvent sa présence dans l'histoire des sciences et sa nécessité dans la science en marche.

En 1835, la $28^{\mathrm{e}}$ leçon du Cours de Comte marque un tournant : elle comporte une importante Théorie fondamentale des hypothèses (p. 433-455), qui ébranle l'image de Comte comme un empiriste étroit, entièrement opposé aux hypothèses (Laudan, 1981, p. 150). Comte l'introduit par un avertissement : il se trouve, dit-il, «obligé de choquer directement des opinions encore très accréditées parmi les physiciens » (p. 431). Il va en effet affirmer, et répéter, que dans les sciences l'introduction des hypothèses est «strictement indispensable », entités que chaque physicien sait pourtant explicitement et catégoriquement exclues par le grand Newton. Comte insiste sur «la fonction fondamentale » de «ce moyen nécessaire ». Son disciple et ami Valat estime qu'il élève trop l'hypothèse en lui assignant le rang de «troisième voie rationnelle de l'esprit, pour arriver à la vérité », en dehors de l'induction et la déduction (1866).

Pour Laudan «il est aisé de sous-estimer l'importance historique de l'adoption des hypothèses par Comte. Dans le contexte de la pensée du début du $19^{\text {ème }}$ siècle, l'insistance répétée de Comte sur le rôle indispensable des conjectures est d'une signification considérable.»(1981, p. 151). Cette importance est occultée par le fait que Comte dit admettre les hypothèses relatives aux lois des phénomènes mais exclure celles, chimériques et non vérifiables, sur leur cause et leur nature intime : bien que, dans les exemples qu'il prend, la limite soit floue (il légitime ainsi l'hypothèse atomique), on a retenu l'éviction plutôt que l'adoption.

Il sera suivi dans cette adoption, mais non dans cette démarcation, par Whewell, qui analyse les démarches en historien des sciences et trace clairement la voie de l'hypothèse au milieu du $\mathrm{XIX}^{\mathrm{e}}$ siècle. Commentant la position de Comte, il proclame que dans bien des cas, «proscrire l'investigation des causes serait anéantir la science »(1840, p. 269). Whewell anticipe les vues de Popper : "Former des hypothèses, puis se donner beaucoup de peine et déployer 
beaucoup d'habileté pour les réfuter (...) est la règle chez ceux qui ont le génie de la découverte » $(1840$, p. 221).

En France, Chevreul (en 1850) réhabilite l'hypothèse avant Claude Bernard (en 1865) qui écrit « un long plaidoyer pour le recours à l'idée dans la recherche, étant entendu qu'une idée scientifique est une idée directrice et non une idée fixe. »(Canguilhem, 1968, p. 233).

Nombreux sont les appels de $\mathrm{Cl}$. Bernard en faveur de celles-ci : «le jour de la dernière hypothèse serait le dernier jour de la science » (Principes, p. 77). Ils eurent pourtant du mal à se faire entendre : des lecteurs de son Introduction à l'étude de la médecine expérimentale (1865) dotés de lunettes empiristes -et parmi ceux-ci, les promoteurs de la leçon de choses-, y lurent que toute recherche débutait par une observation, sans prêter attention au chapitre suivant intitulé "Une recherche expérimentale a pour point de départ une hypothèse ou une théorie », ni au fait que même dans les exemples mettant en avant les observations, celles qui retiennent son attention concernent ce qu'il nomme dans ses notes « un fait qui ne rentre pas dans les idées admises » (cité par Grmek, 1973, p. 33), une «observation polémique » dira plus tard Bachelard (1934, p. 15).

$\mathrm{Au} \mathrm{XX}^{\mathrm{e}}$ siècle, cette troisième voie est consacrée par Popper (1934) et les scientifiques modernes qui le soutiennent. Canguilhem fait en 1942 une mise au point en sa faveur dans ses Leçons sur la méthode : "L'épistémologie contemporaine ne connaît ni les sciences inductives, ni les sciences déductives. (...) Elle ne connaît que des sciences hypothéticodéductives ». Il utilise cette formule marquante : «il faut la raison pour faire une expérience et il faut l'expérience pour se faire une raison. $»^{3}$

\section{L'extension de la nuit des hypothèses sur l'enseignement}

\subsection{La chape inductive}

Si la longue nuit des hypothèses a pris fin dans l'histoire des sciences, elle n'est pas encore achevée dans les pratiques habituelles d'enseignement. Et sans doute peut-on avancer que le triomphe de Newton et l'influence considérable de ses déclarations contre les hypothèses dans les discours des scientifiques ont envahi tout autant le monde de l'enseignement.

Ce sont deux scientifiques de premier plan qui transposeront en classe le mot d'ordre newtonien : Cuvier sous Napoléon I ${ }^{\mathrm{er}}$ puis sous la Restauration, J.-B. Dumas sous Napoléon III. Cuvier déclarait qu'une des qualités les plus précieuses d'un savant était... « sa haine des hypothèses ${ }^{4}$. Il s'oppose à celles de Geoffroy Saint-Hilaire sur le plan d'organisation des animaux et de Lamarck sur le transformisme. Rostand (1945) décrit le débat épistémologique en jeu : «Cuvier représentait le respect du fait, Geoffroy le droit à l'hypothèse » (p. 114). «[Lamarck et Geoffroy] ne s'effarouchent d'aucune supposition; il ne leur coûte rien d'imaginer le passage d'un poisson à un reptile, d'un reptile à un mammifère... Cuvier (...) répugne à de telles conjectures, qui lui paraissent relever de la fable, non de la science » (p. 128).

Dumas énonce ce qui importe dans la méthode : «C'est la foi la plus complète dans le témoignage des sens ; c'est une confiance sans bornes accordée à l'expérience ; c'est une aveugle soumission à la puissance des faits »(1836, p. 3-4). Hulin-Jung relève : «Les conceptions sur la physique du chimiste J.-B. Dumas ont influé sur l'orientation de l'enseignement de cette discipline » $(1989$, p. 310).

\footnotetext{
${ }^{3}$ Canguilhem, G. (1942). Leçons sur la méthode, in Bourdieu, P., Passeron, J.-C. et Chamboredon, J.-C. (1968), Le métier de sociologue : préalables épistémologiques, Mouton de Gruyter, 2005, p. 267-272.

${ }^{4}$ Cuvier, G. (1841). Histoire des sciences naturelles, Fortin, Masson et Cie, tome 2, p. 277 et tome 4, p. 221.
} 
C'est contre les écarts à cette voie s'élevant des faits aux théories que les réformateurs veulent lutter en 1902, ne jurant que par l'induction (Hulin (éd.), 2000 et 2002). C'est un moment important car, comme le signale Hulin (2000, p. 14), ces considérations méthodologiques se feront sentir jusqu'aux temps présents.

En physique, «on doit privilégier la méthode inductive » pour «conduire de l'observation concrète à la Loi », indique encore un rapport datant de... 1989 (Rapport Bergé), à la grande désolation de l'historien Blay (2006) : «Après un siècle, donc, de recherches historiques, d'analyses épistémologiques et philosophiques, les mêmes thèses sont énoncées sans le moindre souci critique et avec la même indigence conceptuelle ; c'est à désespérer ou à se croire entouré de perroquets. » Blay pointe la responsabilité de Newton, par la présentation arrangée de son travail, dans la création d'une "illusion méthodologique" si durable.

En sciences naturelles, une opposition méthodologique intéressante sépare en France les deux inspecteurs généraux des années $1950^{5}$ : pour l'un (Obré), la méthode à suivre est «l'éducation par perception directe et induction continue. ». Mais pour son collègue (Campan), il faut «"armer l'intelligence", suivant l'expression même de Claude Bernard », et passer par «la conception d'hypothèses ». Les élèves «mis en présence d'un problème, il convient de leur laisser le soin de le poser et de chercher à le résoudre ». On voit qu'on est loin de 1" "induction continue" de son collègue, et on constate à la fois la référence à $\mathrm{Cl}$. Bernard et l'apparition de "problèmes" à résoudre. Sans doute pour faire un peu de ménage, une circulaire de $1952^{6}$ préconise le style d'enquête ou de redécouverte, que les deux inspecteurs interprètent aussitôt chacun à sa manière, inductive ou hypothético-déductive : en plein $\mathrm{XX}^{\mathrm{e}}$ siècle, un revival pédagogique des oppositions épistémologiques du $\mathrm{XVII}^{\mathrm{e}}$. De son côté, un autre inspecteur général, Canguilhem, affirme en 1951 devant des enseignants que le seul savoir authentique est « une rectification de l'erreur ».

\subsection{Quand les lumières de l'histoire gagnent les didacticiens... mais pas les classes}

Dans un tel contexte, Hérail (1959) met en scène dans un article deux enseignants : l'un qui se frotte les mains parce qu'il est bien «parti de l'expérience », l'autre qui « ne tombera pas, lui, dans le piège de l'empirisme », car il sait fort bien qu'on n'entreprend pas une expérience sans hypothèse. Hérail montre qu'il n'y a pas moins de dogmatisme à imposer une expérience qu'une théorie, mais que cette forme, qu'il nomme cryptodogmatisme, avance simplement masquée. L'empiriste a pu méditer peu après cette formule frappante de Canguilhem pour bien montrer l'importance des idées directrices : «les progrès d'une science expérimentale n'exigent nullement l'acéphalie des expérimentateurs » (1968, p. 235).

Une autre salve est donnée en 1965 par Gohau. Il est illusoire, explique-t-il, de vouloir que l'expérience présentée à l'élève, ou qu'on lui fait réaliser, le conduise comme par la main vers la lumière de la théorie, car seul le professeur est depuis longtemps hors des ténèbres : «La théorie illumine tant l'expérience de l'arrière que le professeur voit le rapport de l'un à l'autre comme éclatant. Il oublie que l'élève voit l'expérience par sa face "obscure". L'expérience ne conduit directement à la théorie que celui... qui connaît la théorie. » L'importance d'une prise d'initiative authentique par l'élève est soulignée, et l'artifice habituel du faux dialogue dénoncé.

Host (1998) rappelait à la fin de sa vie qu'il menait avec son équipe, dans les années 1970, des travaux qui prenaient en compte «les données épistémologiques (Bachelard, Popper, Canguilhem) » afin de définir des objectifs concernant «la pratique d'une démarche de résolution de problème qui part d'une situation ouverte et comporte une investigation

\footnotetext{
${ }^{5}$ Textes d'Obré et Campan présentés dans L'enseignement des sciences naturelles, IPN, 1956.

${ }^{6}$ Les méthodes de l'enseignement du Second Degré, circulaire du 6 octobre 1952 signée de Charles Brunold.
} 
effective des enfants ». En 1973, les publications simultanées de la traduction française de $L a$ logique de la découverte scientifique de Popper, préfacée par Monod qui y apporte tout son poids, et de l'ouvrage de Grmek sur la méthodologie de $\mathrm{Cl}$. Bernard, éclairent crûment les options inappropriées suivies dans les classes. Ce dernier livre, dira Astolfi, «fonctionne comme un véritable révélateur»(1990, p. 205). Cette même année, un trio de jeunes professeurs (Astolfi, Giordan \& Rumelhard, 1973) se signale par un autre article polémique déclarant que "l'histoire des sciences montre bien qu'il n'existe pas de connaissance réelle si un problème ne s'est pas posé », et préconisant un débat de nature scientifique entre élèves.

Les didacticiens décident donc de «prendre appui sur l'histoire des découvertes scientifiques pour montrer leur écart avec la rigidité d'une démarche stéréotypée » (Astolfi, 1990). Mais, reconnaît Astolfi, cette transformation de l'épistémologie scolaire « est trop souvent chez ceux qui s'en réclament et militent en sa faveur, plus idéologique que véritablement didactique ». Et les changements ne s'opèrent pas dans les classes : Rumelhard (2000) en dresse un bilan sans concession, identifiant «une représentation des savoirs scientifiques dénuée de toute dimension spéculative. L'enseignement des disciplines scientifiques est aujourd'hui essentiellement opératoire et manipulatoire. »

L'insistance sur les problèmes et les activités et l'exclusion des hypothèses fait souvent glisser les enseignants vers des séquences présentant la succession: Observation - Problème Activité - Conclusion. Nous avons proposé, pour caractériser de telles démarches, de les désigner par le sigle OPAC (Cariou, 2007, p. 93-94), qui évoque l'extension de la nuit des hypothèses dans l'enseignement, hypothèses que divers auteurs ont comparé à des flambeaux (Diderot, Bernard, Pasteur...). Ce type de progression, de plus, s'accorde bien avec la peur qu'ont souvent les enseignants des hypothèses des élèves (Vérin, 1998 ; Astolfi, 2002).

Ces divers constats permettent de mesurer l'implantation profonde d'une tradition épistémologique aux racines historiques séculaires, qu'il est nécessaire de prendre en compte dans l'élaboration de stratégies didactiques.

\section{De l'histoire des démarches aux approches didactiques}

Les avantages et les obstacles de l'utilisation didactique de l'histoire des sciences ont été signalés par différents auteurs. Deux numéros thématiques d'Aster, le ${ }^{\circ} 5$, Didactique et histoire des sciences (1987) et le $\mathrm{n}^{\circ} 12$, L'élève épistémologue (1991) livrent de nombreux et intéressants éléments de réflexion et exemples d'approche sur ce thème. Sans pouvoir discuter ici de tous les aspects impliqués, mentionnons dans l'article de Fillon (1991) l'indication de deux écueils importants du point de vue des apprentissages méthodologiques, souvent rencontrés : l'anachronisme (difficulté de respecter les logiques historiques des auteurs) et le mythe de l'explication par les origines (exposition d'une histoire sans ses ruptures ni fourvoiements). Des innovations utilisant l'histoire des sciences ont aussi été élaborées pour favoriser chez les élèves la construction d'une image du fonctionnement de la science proche de l'épistémologie contemporaine, comme celles de Merle (2002) sur la découverte par les Grecs de la sphéricité de la Terre, ou les travaux de C. de Hosson (2005) sur la controverse historique, à partir de dialogues construits à la manière de ceux de Galilée.

Des parallèles entre les conceptions des savants du passé et celles des élèves ont souvent été soulignés, menant à des débats sur la notion de "récapitulation", d'identité ou non entre développement historique et individuel. Des études ont montré des similitudes parfois frappantes mais d'autres fois trompeuses, ainsi des étudiants modernes et certains savants du $\mathrm{XVII}^{\mathrm{e}}$ siècle portent en apparence le même regard dubitatif sur l'intérieur du tube de l'expérience de Torricelli, mais pour des raisons que l'analyse révèle différentes (de Hosson \& Caillarec, 2009). Cependant, d'une manière générale, estime Gohau (2006), «la récapitulation fonctionne à la condition de ne pas la prendre dans un sens trop strict». 
L'histoire des sciences peut donc préparer l'enseignant à l'expression des conceptions des élèves, ce qui faisait dire à $\mathrm{F}$. Balibar (1994) que cela pouvait au moins servir à ne pas réprimer, «au nom du prétendu bon sens, telle ou telle conception spontanée développée par tel ou tel élève ».

Les données particulières que nous avons mises en avant permettent d'envisager des pistes complémentaires aux précédentes pour à la fois tenter de contrecarrer "l'épistémologie spontanée" des enseignants et donner aux élèves une image plus authentique des cheminements en science :

- sous l'angle de l'utilisation de textes historiques : comment pourrait-on entrevoir un autre rapport à l'histoire des sciences, à la fois critique et éclairant sur les démarches ?

- sous l'angle de la mise en œuvre des séquences : comment y favoriser le jeu des hypothèses et de leur contrôle, pour contrer les effets encore sensibles de la tradition épistémologique newtonienne?

\subsection{Analyser les démarches tortueuses plutôt qu'exalter les « découvertes »}

L'accent est actuellement mis, en France, à travers le renouveau des programmes du secondaire et des concours d'enseignement, sur l'intégration d'éléments d'histoire des sciences. Cette attention pourrait être développée dans le sens d'une analyse des rapports réciproques entre raison et expérience.

Mais bien souvent, les exemples choisis sont extraits de leur contexte à un point qui leur ôte cette vertu : qu'apporte par exemple une reproduction en classe de l'expérience du foie lavé de $\mathrm{Cl}$. Bernard, «parachutée » sans lien avec son idée directrice erronée (celle d'une destruction du sucre par les animaux qui n'en produisent pas), sans justification, le plus souvent, de son introduction, et sans possibilité pour les élèves d'en faire aisément une interprétation correcte ? On "lave" le foie et on récolte du sucre, on le laisse dans un coin et on le relave, on récolte encore du sucre : tout n'était donc pas venu au premier lavage... Aller de cette expérience de classe à la fonction glycogénique du foie paraît un saut bien audacieux. L'apport n'est donc ici ni conceptuel, ni méthodologique. Le savant est cependant présenté comme l'éminent personnage qui, de l'expérience, extrait directement le concept scientifique.

Canguilhem résume bien le basculement qui est en jeu : "L'histoire d'une science ne saurait être une simple collection de biographies, ni à plus forte raison un tableau chronologique agrémenté d'anecdotes. Elle doit être aussi une histoire de la formation, de la déformation et de la rectification de concepts scientifiques. » (1968, p. 235).

Mais, plus souvent qu'à la déformation des concepts, nous assistons, dans les manuels scolaires, à celle... de l'histoire elle-même, par une extraction d'épisodes déracinés de leur contexte qui interdit de saisir la démarche suivie, les pistes infructueuses et les obstacles surmontés, quand elle n'est pas réarrangée au profit d'une présentation empiriste.

Or le professeur peut faire travailler les élèves sur des textes qui leur permettent de suivre au moins partiellement les cheminements des auteurs dans ces broussailles dont parlait Kepler, les amenant à conclure, comme lui : «Les erreurs nous montrent le chemin de la vérité ».

À titre d'exemple, nous proposons de mettre en regard la manière dont les travaux historiques sur la neurotransmission sont narrés dans un manuel de lycée et les autres choix que pourrait opérer un enseignant plus soucieux de faire pressentir à ses élèves la nature des démarches en sciences.

\footnotetext{
${ }^{7}$ Hon, G. (1987). “On Kepler's Awareness of the Problem of Experimental Error”. Annals of Science, Vol. $44, n^{\circ} 6$, Novembre 1987 , p. 545-591.
} 
La figure 1 correspond à la relation par un manuel actuel de 1re scientifique (en France) de la découverte par Loewi en 1921 de ce que l'on nommera plus tard les neurotransmetteurs. Dans la reconstruction qui en est proposée, le début du second paragraphe retient particulièrement notre attention :

\section{Une expérience historique}

\section{La transmission synaptique}

En 1921, le pharmacologue Otto Loewi réalise une expérience concernant la transmission synaptique. Ayant prélevé le cœur d'une grenouille avec son innervation parasympathique (vous avez vu en Seconde que le nerf vague est capable de diminuer la fréquence des battements du cœur), il le place sous perfusion d'une solution saline et constate qu'il continue à battre normalement. Il prélève alors le cœur d'une seconde grenouille, cette fois sans ses nerfs, et le plonge dans cette solution saline. Là encore, le cœur bat normalement. Il stimule le nerf vague associé au premier cœur : ses battements ralentissent. Il le sort alors de la solution saline, y place le second cœur qui se met à ralentir en dehors de toute stimulation nerveuse...

Loewi formule alors l'hypothèse qu'une substance chimique, le Vagusstoff ou substance vagale, résultant de la stimulation du nerf parasympathique du premier cœur, a été libérée dans le liquide de perfusion. Il lui faudra

Figure 1. Relation de la démarche de Loewi

(Sciences de la Vie et de la Terre, $1^{\text {re }}$ S, Nathan, 2001, p. 208).

«Loewi formule alors 1'hypothèse »: celle-ci paraît n'intervenir qu'après plusieurs expériences, en contradiction flagrante avec la réalité historique, puisque Loewi a émis son hypothèse... 17 ans avant, ainsi qu'il le narre lui-même (1960) : " une expérience permettant de déterminer si oui ou non l'hypothèse de la transmission chimique que j'avais formulée dixsept ans auparavant était correcte. »

L'élève ne sait pas, dans ce récit, pourquoi le chercheur prélève un cœur, puis un autre, l'un avec nerf et l'autre sans, et pas davantage pourquoi le second cœur remplace le premier dans la solution. Quand l'hypothèse paraît, elle ne sert de fil directeur à aucune expérience : seul le nom de la substance paraît importer. Le lien expériences-découvertes paraît direct, comme d'ailleurs le suggère le titre même donné à cet encadré. Une narration en conformité avec ce que vit l'élève par ailleurs quand, en arrivant en classe, on lui fait faire une série d'observations ou d'expériences dont il ne connaît pas le sens, mais d'où jaillira la "découverte".

Mais il se trouve que la suite de l'histoire de la transmission synaptique nous fournit des indications précieuses sur certains aspects psychologiques des démarches à mettre en œuvre en classe. Après ces travaux qui lui ont valu le prix Nobel en 1936, Loewi soutient en effet que la transmission synaptique est chimique, contre Eccles qui la pense électrique. Eccles suit « les idées conventionnelles sur la recherche scientifique »: « selon la vision inductive de la science (...) c'est un signe de faillite, hautement regrettable, lorsqu'un scientifique épouse une hypothèse qui est réfutée $»^{8}$.

Mais Eccles se rend bientôt compte qu'il est dans ce cas, ce qui le plonge dans « un état d'extrême dépression ». Heureusement, un changement d'état d'esprit, relativement à sa

\footnotetext{
${ }^{8}$ Cet extrait de Eccles et les suivants sont cités dans Debru, C. (1987). Philosophie moléculaire. Monod, Wyman, Changeux. Vrin, p. 184-185.
} 
vision de la recherche scientifique, s'opère bientôt chez Eccles : «Ce fut à cette époque que je rencontrai Popper. Parmi les choses importantes que j'appris de lui, la plus importante peutêtre fut qu'il n'était pas déshonorant de voir ses hypothèses favorites réfutées. Ce fut la meilleure nouvelle que j'aie eue depuis longtemps. Popper me persuada, en fait, de formuler mes hypothèses électriques de la transmission synaptique excitatrice et inhibitrice avec suffisamment de précision et de rigueur pour susciter une réfutation expérimentale stricte : et ce fut ce qui leur arriva quelques années plus tard ».

Eccles se rallie à l'hypothèse adverse, poursuit ses travaux sur la synapse, et reçoit finalement pour ceux-ci le prix Nobel en 1963. Sa biographie Nobel commente : «Il a le sentiment que cette relation aux hypothèses a non seulement accru son pouvoir conceptuel, mais l'a aussi grandement aidé émotionnellement ! Il peut désormais se réjouir même de la réfutation d'une théorie chérie, car cela aussi est un succès scientifique. »

Quelle leçon! Nos élèves sont justement, généralement, dans le premier état d'esprit d'Eccles : on voit tout l'effet psychologique possible d'insister non sur l'exactitude a priori d'une hypothèse, mais seulement sur sa recevabilité et sa cohérence. Tomber sur la bonne solution n'a pas une importance cruciale et n'est pas davantage valorisable que proposer une solution logique, qui peut-être sera fausse : on ne peut pas savoir puisque, justement, on cherche ! Celui qui "avait juste", Loewi se retrouve avec le prix Nobel, mais celui qui "avait faux", Eccles... aussi !

Toutes les grandes notions issues des travaux de neurophysiologie de Loewi et Eccles sont au programme de la série scientifique du lycée : neurotransmetteurs, réflexe myotatique, PPS. À l'inverse de la présentation historique du type de celle du manuel scolaire ci-dessus, qui montre un chercheur allant droit au but par ses multiples expériences, on peut mesurer tout l'intérêt qu'il y aurait, pour l'état d'esprit des élèves, à porter à leur connaissance le désespoir d'Eccles et la manière dont il se releva de son hypothèse «mise au rencart », sort que pourraient aussi subir les leur pour peu qu'on favorise leur expression dans les séquences de classe.

\subsection{Ranimer en classe le flambeau des hypothèses}

$\mathrm{Cl}$. Bernard voyait, dans l'hypothèse, la flamme indispensable pour sortir de l'opacité empiriste : «Sans l'hypothèse et la théorie qui sont les flambeaux qui dirigent l'homme, (...) on reste dans un obscur empirisme. » (Principes, p. 226).

Reconnaître dans les classes le rôle éminent des hypothèses et favoriser leur jeu, comme ce fut le cas dans les sciences elles-mêmes après leur longue disgrâce, constitue une autre approche possible pour faire pénétrer les élèves dans des rouages révélateurs de la nature de la science.

Un moyen d'y parvenir est de fonder certaines séquences sur les propositions des élèves, en mettant en place de conditions telles qu'ils puissent élaborer des hypothèses, les soumettre au débat en classe et soupeser leur pertinence, en concevoir des moyens de contrôle, eux-mêmes débattus, et les rejeter ou non après leurs mises à l'épreuve.

Dans cette optique, nous avons mené en France une recherche didactique dans le cadre d'une thèse portant sur la formation de l'esprit scientifique des élèves (2006-2008) avec une équipe d'enseignants de SVT de collège et de lycée (20 classes, de la $5^{\mathrm{e}}$ à la $1^{\mathrm{re}}$ scientifique) désireux de mettre en œuvre dans leurs classes des séquences d'investigation plus authentiques, en termes de démarches, par leur plus grande conformité avec les données épistémologiques et historiques, et privilégiant l'initiative des élèves.

Notre propos n'est pas de présenter ici cette recherche dans ses détails méthodologiques et ses résultats (nous renvoyons le lecteur intéressé à la présentation de cette étude dans le numéro 1 
de la revue RDST (Cariou, 2010) ainsi qu'à la thèse elle-même, consultable en ligne ${ }^{9}$ ) : nous souhaitons simplement proposer un outil issu de ces travaux, une " échelle d'authenticité » tournée vers la réhabilitation de la voie des hypothèses.

Des séquences respectant un certain nombre de principes destinés à en avant les propositions d'hypothèses et de stratégies de contrôle par les élèves ont été présentées aux enseignants, qu'il leur était loisible d'adopter, d'adapter ou de remplacer par d'autres à la condition que ces principes, considérés comme des « critères d'authenticité » des démarches d'investigation, soit respectés. Les résultats obtenus au moyen de tests et de questionnaires ont permis de constater un développement de l'esprit créatif des élèves, en jeu dans l'émission d'hypothèses, ainsi que de leur esprit de contrôle.

Ultérieurement, les critères retenus ont été formalisés et listés dans une échelle d'authenticité, dans l'esprit de celle élaborée par Schwab pour déterminer des niveaux d'“ouverture et permissivité" dans les séquences de sciences étiquetées inquiry (1962, p. 55). Ils apparaissant dans le tableau 1 (C1 à C10).

Sont ainsi considérés comme des gages d'authenticité le fait $(\mathrm{C} 1)$ que le problème présente un caractère énigmatique mais soit à portée des élèves et (C2) leur soit réellement posé ; que (C3) les hypothèses proviennent d'eux, (C4) qu'un débat entre eux sur leur recevabilité soit instauré et que (C5) celles retenues aient un aspect douteux; que (C6) les activités soient conçues par eux afin de les éprouver, (C7) qu'ait lieu un débat sur leur pertinence de ces mises à l'épreuve et $(\mathrm{C} 8)$ qu'aucune ne porte sur des faits évidents ni ne soit sans lien direct avec le fil conducteur de l'investigation; que (C9) une nouvelle phase de débat s'ouvre au moment d'interpréter les résultats obtenus relativement aux hypothèses émises, enfin que (C10) les conclusions soient élaborées par les élèves et non dictées.

\begin{tabular}{|c|c|c|c|}
\hline \multicolumn{3}{|c|}{$\begin{array}{l}\text { Critères d'Authenticité des Démarches d'Investigation } \\
\text { (Échelle CADI) En grisé : phases de débat scientifique }\end{array}$} & \\
\hline C1 & $\begin{array}{l}\text { Qualité } \\
\text { du problème }\end{array}$ & $\begin{array}{l}\text { Problème représentant, pour les élèves, une énigme, un obstacle, une } \\
\text { rupture, une "morsure" (Dewey) }\end{array}$ & 1 \\
\hline $\mathrm{C} 2$ & $\begin{array}{l}\text { Raison d'être } \\
\text { du problème }\end{array}$ & $\begin{array}{l}\text { Problème mobilisant les forces intellectuelles des élèves, pour être résolu à } \\
\text { partir de leurs propositions }\end{array}$ & 1 \\
\hline C3 & $\begin{array}{l}\text { Origine des } \\
\text { Hypothèses }\end{array}$ & $\begin{array}{l}\text { Hypothèse(s) venant d'élèves, traduisant leur vision, reflétant leurs } \\
\text { conceptions }\end{array}$ & 1 \\
\hline C4 & $\begin{array}{l}\text { Examen des } \\
\text { Hypothèses }\end{array}$ & $\begin{array}{l}\text { Discussion par les élèves de la recevabilité des hypothèses (critères de } \\
\text { recevabilité : lien avec le problème, cohérence avec les acquis...) }\end{array}$ & 1 \\
\hline C5 & $\begin{array}{l}\text { Qualité des } \\
\text { hypothèses } \\
\text { retenues }\end{array}$ & $\begin{array}{l}\text { Hypothèses retenues portant sur des faits encore inconnus, dont la } \\
\text { connaissance aiderait à résoudre le problème }\end{array}$ & 1 \\
\hline C6 & $\begin{array}{l}\text { Origine des } \\
\text { activités }\end{array}$ & $\begin{array}{l}\text { Activité(s) pré-méditées, conçues, demandée(s) par les élèves** } \\
\text { (observations, expériences ou documents montrant si...) }\end{array}$ & 1 \\
\hline C7 & $\begin{array}{l}\text { Examen des } \\
\text { activités } \\
\text { proposées }\end{array}$ & $\begin{array}{l}\text { Phase de discussion par les élèves de la pertinence des activités proposées } \\
\text { (s'agit-il de conséquences déduites des hypothèses ?) }\end{array}$ & 1 \\
\hline C8 & $\begin{array}{l}\text { Qualité des } \\
\text { activités } \\
\text { retenues }\end{array}$ & $\begin{array}{l}\text { Activités dont les résultats attendus apporteront des éléments nouveaux } \\
\text { utiles à la résolution du problème }\end{array}$ & 1 \\
\hline
\end{tabular}

\footnotetext{
${ }^{9}$ Former l'esprit scientifique en privilégiant l'initiative des élèves dans une démarche s'appuyant sur l'épistémologie et l'histoire des sciences, thèse de doctorat en Sciences de l'Éducation, dir. A. Giordan et J. Guichard, soutenue le 29 janvier 2009, université de Genève, http://tel.archives-ouvertes.fr/tel-00521174/fr/ (consulté le 10 février 2011).
} 


\begin{tabular}{|c|c|l|c|}
\hline C9 & $\begin{array}{l}\text { Discussion des } \\
\text { interprétations }\end{array}$ & $\begin{array}{l}\text { Phase de discussion entre élèves de leurs interprétations des résultats } \\
\text { obtenus }\end{array}$ & 1 \\
\hline C10 & $\begin{array}{c}\text { Origine des } \\
\text { conclusions }\end{array}$ & $\begin{array}{l}\text { Conclusions établies par les élèves, admises et généralisées sous le contrôle } \\
\text { du professeur }\end{array}$ & 1 \\
\hline \multicolumn{3}{|r|}{ Niveau d'investigation =-> } & \\
\hline
\end{tabular}

Tableau 1. Échelle d'authenticité à 10 niveaux pour démarches d'investigation

Les critères correspondant à des débats entre élèves (en grisé dans le tableau $1: \mathrm{C} 1, \mathrm{C} 4, \mathrm{C} 7$, C9 et C10) permettent d'instaurer le « débat scientifique dans la classe » que souhaitent Johsua et Dupin, avec les modalités qu'ils décrivent, notamment concernant l'expression d'hypothèses sous-tendues par des conceptions implicites (1993, p. 335-336).

La recevabilité des hypothèses proposées comme les jugements sur la pertinence des tests suggérés ne dépendent pas du professeur, mais d'échanges argumentés entre élèves.

Le respect de ces principes a permis d'éviter certains des travers habituels, qui tous conduisent à un amoindrissement ou à un effondrement du rôle des hypothèses : problème posé au tableau, mais non aux élèves, ou dont la solution est déjà connue; absence d'hypothèses, ou venant de l'enseignant, ou portant sur des faits déjà connus; activités "parachutées" avec ou sans lien avec le problème; conclusions dictées. Sont alors mises en œuvre dans les classes des séquences dans lesquelles les élèves échafaudent un éventail d'hypothèses, souvent inexactes, comme dans la recherche scientifique.

Cette approche permet en outre d'utiliser des éléments historiques pour valoriser des élèves qui avancent des propositions fausses, mais recevables au vu des connaissances de la classe (critère C4) : l'enseignant peut mentionner qu'une idée avancée rejoint celle de tel savant prestigieux de telle époque qui, au stade où il en était lui aussi, ne pouvait pas non plus savoir. Un élève peut également se trouver valorisé lorsqu'il propose par exemple une expérience jugée pertinente par la classe (critère $\mathrm{C7}$ ), et qu'un document historique peut être fourni montrant que des savants ont procédé de la manière proposée, que leurs expériences aient conduit à résoudre le problème ou pas. On peut ainsi ne leur fournir un document relatant les expériences de Réaumur sur la digestion qu'en réponse à la proposition de placer “ce qu'il y a dans le tube digestif" sur des aliments, comme test de l'hypothèse d'une "attaque" par des substances produites à ce niveau.

L' « échelle d'authenticité » telle que la présente le tableau 1 peut être utilisée, en attribuant 0 ou 1 point pour chaque critère selon qu'il paraît respecté ou non, pour estimer le niveau d'investigation une séquence. Nous proposons cet outil, issu de notre recherche où le respect de ces critères a redonné aux hypothèses un rôle similaire en classe à celui qu'elles jouent dans l'histoire des sciences, comme une aide pour un enseignant désireux, lorsqu'il prépare sa séquence ou au moment où il y revient, d'accroître ce rôle et, en visant l'instauration en classe $\mathrm{du}$ « jeu des possibles » cher à François Jacob, de les extraire de l'éteignoir empiriste.

Le regard jeté sur les discours historiques concernant les méthodes nous a permis de dégager les séquelles des sentences de Newton contre l'usage des hypothèses dans la recherche et, par répercussion, dans l'enseignement des sciences qui de nos jours, même lorsqu'il cherche à prendre appui sur l'histoire des sciences, en conserve encore les traces.

Si l'introduction d'éléments historiques dans les programmes vise autre chose qu'une illustration agréable par la présentation d'augustes savants en costume d'époque, si elle vise notamment une meilleure compréhension par les élèves des itinéraires scientifiques, une 
sensibilisation des enseignants à l'histoire des démarches nous paraît utile pour qu'ils puissent à la fois opérer des choix de textes présentant aux élèves les cheminements effectifs des chercheurs, et pour leur permettre d'en vivre de semblables, toutes proportions gardées, dans les classes, les deux types d'approches des démarches se complétant: mieux les suivre dans l'histoire, et mieux les vivre en classe.

\section{Références}

APOSTOLOU, A. \& KOULAIDIS, V. (2010). Epistemology and science education: a study of epistemological views of teachers. Research in Science \& Technological Education, 28(2), 149-166.

ASTOLFI, J.-P. (1990). «L'émergence de la didactique de la biologie, un itinéraire ». Aster n 11.

ASTOLFI, J.-P. (2002). «L'œil, la main, la tête ». Expérimenter, Cahiers pédagogiques $n^{\circ} 409$.

ASTOLFI, J.-P., GIORDAN, A. et RUMELHARD, G. (1973). Pourquoi l'autonomie des élèves en biologie ? A.P.B.G. $\mathrm{n}^{\circ} 4-1973$.

BACHELARD, G. (1932). « Noumène et microphysique », in Études, Vrin, 2002.

BACHELARD, G. (1934). Le nouvel esprit scientifique, PUF, 1987.

BACON, F. (1605). Du progrès et de la promotion des savoirs, Gallimard, 1991.

BACON, F. (1620). Novum Organum, PUF, 1986.

BALIBAR, F. (1994). L'histoire des sciences, une école de pensée critique. In B. Andries et I. Beigbeder (Coord). La culture scientifique pour les professeurs des écoles, CNDP, Hachette Éducation, p. 37-42.

BERNARD, C. (1865). Introduction à l'étude de la médecine expérimentale, Garnier-Flammarion, 1966.

BERNARD, C. Principes de médecine expérimentale (rédigés de 1862 à 1877), $2^{\circledR}$ éd. PUF 1987.

BLAY, M. (2006). Concepts, faits scientifiques et théories, Raison Présente n 157-158, décembre 2006.

CANGUILHEM, G. (1968) Études d'histoire et de philosophie des sciences, Vrin, 1994.

CARIOU, J.-Y. (2007). Faire vivre des démarches expérimentales. Delagrave.

CARIOU, J.-Y. (2010). Les opinions vulnérables, tremplin vers le savoir. RDST-Recherches en Didactique des Sciences et des Technologies, $\mathrm{n}^{\circ} 1$.

CLÉMENT, P. (1998). «La biologie et sa didactique, dix ans de recherche », Aster $\mathrm{n}^{\circ} 27$.

COHEN, I. B. (1960). Les origines de la physique moderne, Seuil, 1993.

COMTE, A. (1830). Cours de philosophie positive, tome 1, Bachelier.

COMTE, A. (1835). Cours de philosophie positive, tome 2, Bachelier.

DEMOUNEM, R. \& ASTOLFI, J.-P. (1996). Didactique des sciences de la vie et de la Terre. Nathan.

DESCARTES, R. (1637). Discours de la Méthode. GF-Flammarion, 2000.

DESCARTES, R. (1644). Principes de la philosophie. In Euvres philosophiques, III, F. Alquié, Garnier, 1998.

DORIER, J.-L. (2000). Recherches en histoire et en didactique des mathématiques sur l'algèbre linéaire Perspective théorique sur leurs interactions. Cahier du laboratoire Leibniz, $\mathrm{n}^{\circ} 12$.

DRAKE, E. T. (1996). Restless Genius Robert Hooke and His Earthly Thoughts, Oxford University Press.

DUHEM, P. (1906) La théorie physique, Vrin 1997.

DUMAS, J.-B. (1836). Leçons sur la philosophie chimique professées au Collège de France, Bechet jeune, 1837.

ELLENBERGER, F. (1994). Histoire de la Géologie, tome 2, Tech. \& Doc./Lavoisier.

FILLON P. (1991). « Histoire des sciences et réflexions épistémologiques des élèves. » Aster $\mathrm{n}^{\circ} 12$.

GIL-PÉREZ, D., VILCHES, A., FERNANDEZ, I., CACHAPUZ, A., PRAIA, J., VALDES, P, \& SALINAS, J. (2005). Technology as"Applied Science': a Serious Misconception that Reinforces Distorted and Impoverished Views of Science. Science \& Education, 14, 309-320.

GOHAU, G. (1965). «Valeur et place de l'expérience ». Biologie Géologie, bulletin APBG n³-1965.

GOHAU, G. (1977). «Difficultés d'une pédagogie de la découverte dans l'enseignement des sciences ». Aster n5, INRP, 1987.

GOHAU, G. (2006). Enseigner l'histoire des sciences, pourquoi ? Cahiers Rationalistes n 580 , janvier-février 2006.

GRMEK, M. (1973) Raisonnement expérimental et recherches toxicologiques chez Claude Bernard, Droz.

GUICCIARDINI, N. (2003). Newton, l'horloger du monde. «Les génies de la science », Ed. Pour la Science, $11 / 2003$.

HÉRAIL, P. (1959). "Méthode expérimentale et cryptodogmatisme ”, Bull. Union des Naturalistes, 3-1959.

HOSSON (de) C. (2005). La controverse historique : un outil didactique (partie 2), in Bulletin de l'Union des Physiciens, ${ }^{\circ} 870$.

HOSSON (de), C. et CAILlaREC, B. (2009). L'expérience de Blaise Pascal au Puy de Dôme : analyse des difficultés des étudiants de premier cycle universitaire et confrontation historique. Didaskalia $\mathrm{n}^{\circ} 34$.

HOST, V. (1998). Évolution de l'enseignement scientifique en France depuis un siècle, présenté par Jeannine Deunff et Jean-Michel Host, <http://www.paysdelaloire.iufm.fr/IMG/pdf/host.pdf> consulté le 20 octobre 2008. 
HULIN, N. (dir., 2000). Physique et «humanités scientifiques». Autour de la réforme de l'enseignement de 1902. Études et documents. Septentrion.

HULIN, N. dir. (2002) Sciences naturelles et formation de l'esprit. Autour de la réforme de l'enseignement de 1902. Presses Universitaires du Septentrion.

HULIN-JUNG, N. (1989). L'organisation de l'enseignement des sciences, Ed. du Comité des Travaux historiques et scientifiques.

JOHSUA S. \& DUPIN J.-J. (1993) Introduction à la didactique des sciences et des mathématiques, PUF.

KAHN, P. (2002). L'enseignement des sciences naturelles entre philosophie et pédagogie. In Nicole Hulin (éd.), Sciences naturelles et formation de l'esprit. Autour de la réforme de l'enseignement de 1902. Presses Universitaires du Septentrion.

KANT, I. (1781, $2^{\mathrm{e}}$ éd. 1787). Critique de la raison pure, PUF, 1986.

KOLLERSTROM, N. (1999). "How Newton Failed to Discover the Law of Gravity", Annals of Science, 59, p. 331-356.

KOYRÉ, A. (1968) Études newtoniennes. Gallimard.

KUHN, T. S. (1958). Introduction à Cohen, I. B. (ed., 1958), Isaac Newton's Papers and Letters on Natural Philosophy, Harvard University Press.

LAUDAN, L. (1981). Science and hypothesis, Reidel.

LEDERMAN, N. G. (2007). Nature of Science: Past, Present, and Future. In Abell, S. K. and Lederman, N. G. (eds.). Handbook of Research on Science Education. Mahwah : Lawrence Erlbaum Associates, p. 831-880.

LOEWI, O. (1960) «An Autobiographic Sketch », Perspect. Biol. Med. 4, 3-25.

MEDAWAR, P.B. (1967). The Art of the Soluble, Methuen \& Co Ltd.

MERLE, H. (2002). Histoire des sciences et sphéricité de la Terre : compte rendu d'innovation. Didaskalia $\mathrm{n}^{\circ} 20$.

NEWTON, I. (1687, 1713, 1726). Principes mathématiques de la philosophie naturelle, trad. E. du Châtelet, tome II, Desaint \& Saillant, 1759.

POPPER, K. (1934). La logique de la découverte scientifique, Payot, 1989.

ROBARDET, G., \& GUILLAUD, J.-C. (1997). Éléments de didactique des sciences physiques, PUF.

ROHAULT, J. (1671). Traité de physique. Paris : Veuve de Ch. Savreux.

ROSTAND, J. (1945) Esquisse d'une histoire de la biologie, Gallimard, 1973.

RUMELHARD, G. (2000) «Sciences de la vie, philosophie, sciences humaines ». Aster $n^{\circ} 30$, INRP.

SCHWAB, J. J. (1962). "The teaching of science as inquiry". In Schwab, J.J. and Brandwein, P.F. (Eds, 1962).

The teaching of science, Harvard University Press.

VALAT, J.-P. (1866). «Des hypothèses dans la science», Actes de l'Académie nationale des sciences, belleslettres et arts de Bordeaux, E. Dentu, 1866, p. 613-640.

VÉRIN, A. (1998). «Enseigner de façon constructiviste, est-ce faisable ? », Aster $\mathrm{n}^{\circ} 26$.

WHEWELL, W. (1840). The Philosophy of the Inductive Sciences, Founded Upon Their History, Vol. 2, Parker. 